\title{
Robo2 determines subtype-specific axonal projections of trigeminal sensory neurons
}

\author{
Y. Albert Pan ${ }^{1, \pm}$, Margaret Choy ${ }^{1,2}$, David A. Prober ${ }^{1, *}$ and Alexander F. Schier ${ }^{1,3,4,5, \pm}$
}

\begin{abstract}
SUMMARY
How neurons connect to form functional circuits is central to the understanding of the development and function of the nervous system. In the somatosensory system, perception of sensory stimuli to the head requires specific connections between trigeminal sensory neurons and their many target areas in the central nervous system. Different trigeminal subtypes have specialized functions and downstream circuits, but it has remained unclear how subtype-specific axonal projection patterns are formed. Using zebrafish as a model system, we followed the development of two trigeminal sensory neuron subtypes: one that expresses trpa1b, a nociceptive channel important for sensing environmental chemicals; and a distinct subtype labeled by an is/et1 reporter (Is/1SS). We found that Trpa1b and Is/1SS neurons have overall similar axon trajectories but different branching morphologies and distributions of presynaptic sites. Compared with Trpa1b neurons, Is/1SS neurons display reduced branch growth and synaptogenesis at the hindbrain-spinal cord junction. The subtype-specific morphogenesis of Is/1SS neurons depends on the guidance receptor Robo2. robo2 is preferentially expressed in the Is/1SS subset and inhibits branch growth and synaptogenesis. In the absence of Robo2, Is/1SS afferents acquire many of the characteristics of Trpa1b afferents. These results reveal that subtypespecific activity of Robo2 regulates subcircuit morphogenesis in the trigeminal sensory system.
\end{abstract}

KEY WORDS: Nociception, Somatosensory, TRPA1, Targeting, Axon guidance, Synaptogenesis, Zebrafish

\section{INTRODUCTION}

The remarkable diversity and specific connectivity of sensory neurons are crucial for the ability to sense and distinguish environmental stimuli (Kay et al., 2011; Luo and Flanagan, 2007; Marmigere and Ernfors, 2007; Mombaerts et al., 1996). In the somatosensory system, chemical, mechanical and thermal stimuli to the head are sensed by different trigeminal sensory neuron subtypes that have varied morphologies and distinct axonal connections to second-order neurons (Brodal, 2010; Erzurumlu et al., 2010; Todd, 2010). Trigeminal sensory neuron subtypes can be characterized by several molecular criteria, such as the expression of high-affinity neurotrophin receptors ( $\operatorname{trk} A, \operatorname{trk} B$ and $\operatorname{trk} C$; also known as ntrk1, ntrk2 and ntrk3, respectively), transcription factors, neuropeptides, ion channels and $G$ protein-coupled receptors (Basbaum et al., 2009; Liu and Ma, 2011; Woolf and Ma, 2007). Examples include transient receptor potential (TRP) ion channels that confer sensitivity to temperature and chemicals (TRPV1 for heat and TRPA1 for noxious chemicals), and P2X class ion channels that detect ATP and modulate pain sensation (Caterina et al., 1997; Chen et al., 1995; Story et al., 2003). These markers are conserved in vertebrates and allow specific labeling of genetically defined sensory subtypes, enabling the study of specific

\footnotetext{
${ }^{1}$ Department of Molecular and Cellular Biology, Harvard University, 16 Divinity Avenue, Cambridge, MA 02138, USA. ²Developmental Genetics Program, New York University School of Medicine, New York, NY 10016, USA. ${ }^{3}$ Harvard Stem Cell Institute, Harvard University, Cambridge, MA 02138, USA. ${ }^{4}$ Broad Institute of MIT and Harvard, Cambridge, MA 02142, USA. ${ }^{5}$ Center for Brain Science, Harvard University, Cambridge, MA 02138, USA.

*Present address: Division of Biology, California Institute of Technology, Pasadena, CA 91125, USA

‡Authors for correspondence (yapan@mcb.harvard.edu; schier@fas.harvard.edu)
}

Accepted 1 December 2011 sensory subcircuits (Caron et al., 2008; Cavanaugh et al., 2011; Dhaka et al., 2008; Kucenas et al., 2006; Takashima et al., 2007; Zylka et al., 2005).

Afferent morphologies of different trigeminal subtypes share several features. Each trigeminal sensory neuron extends a single axon shaft along the lateral white matter of the hindbrain and spinal cord. Numerous medially projecting branches are later formed and innervate a series of target nuclei along the anteroposterior axis of the hindbrain and spinal cord (Erzurumlu et al., 2006; Jacquin et al., 1986). The anteriorly located principal sensory nucleus (PrV) is the main relay station for mechanical stimuli, whereas the posteriorly located spinal trigeminal nucleus $(\mathrm{SpV})$ and cervical spinal dorsal horn are important for sensing noxious and thermal stimuli (Brodal, 2010; Noma et al., 2008). Functional specificity is determined by the spatial pattern of branch termination, which differs greatly between subtypes (Erzurumlu et al., 2010; Marmigere and Ernfors, 2007). For example, axon terminals that express TRPA1 and P2x3 (also known as P2rx3) are sparse in PrV and dense in SpV (Kim et al., 2008; Kim et al., 2010). Despite the importance of accurate subcircuit formation for the proper transmission of sensory information, it has remained unclear how different trigeminal sensory neuron subtypes select specific targets along the anterior-posterior axis.

One possible mechanism for selective anterior-posterior targeting is through regulation of branch growth and synaptogenesis by Robo/Slit signaling. Robo proteins are cell surface receptors that bind to the secreted ligand Slit. Signaling via Robo receptor activation plays diverse roles in shaping the developing nervous system, including axon targeting, synaptogenesis and cell migration (Campbell et al., 2007; Cho et al., 2007; Cho et al., 2011; Dickson and Gilestro, 2006; Xiao et al., 2011). Robo/Slit signaling can exert either positive or negative influences on axonal growth and branching, in some cases having both effects on the same cell (Ma and Tessier-Lavigne, 2007; Ypsilanti et al., 2010). Previous reports, however, are conflicting regarding how Robo/Slit signaling affects 
somatosensory primary afferents. In rats, exogenously supplied Slit2 can promote growth and branching of trigeminal afferents (Ozdinler and Erzurumlu, 2002). By contrast, genetic studies in zebrafish and mice suggest that Robo activation acts to repel trigeminal afferent branches (Ma and Tessier-Lavigne, 2007; Yeo et al., 2004). For example, Yeo et al. (Yeo et al., 2004) found that overexpression of Slit is sufficient to repel trigeminal afferents in zebrafish embryos, but it has been unclear whether Slit/Robo signaling is necessary for normal trigeminal morphogenesis. Furthermore, it is unclear whether Robo signaling plays a role in regulating the formation of subtypespecific projections.

To determine how subtype-specific axonal projections are formed, we used zebrafish (Danio rerio) trigeminal sensory neurons as a model system. Zebrafish larvae are small, transparent and contain only $\sim 60$ trigeminal sensory neurons per ganglion, making it possible to observe axonal morphogenesis in vivo at single-cell resolution (Caron et al., 2008; Knaut et al., 2005; Sagasti et al., 2005). Using this system, we defined two trigeminal sensory neuron subtypes with distinct afferent morphologies and projection patterns and discovered that Robo2 regulates the development of subtype-specific afferent projections by inhibiting branch growth and synaptogenesis. These results reveal that Robo2 function is essential for subcircuit morphogenesis in the somatosensory system.

\section{MATERIALS AND METHODS}

\section{Zebrafish strains}

Embryos and larvae were raised at $28.5^{\circ} \mathrm{C}$ in water containing $0.1 \%$ Methylene Blue hydrate (Sigma, St Louis, MO, USA). At 24 hours postfertilization, embryos were transferred to water containing $0.003 \% 1$ phenyl-2-thiourea (PTU; Sigma) to prevent pigment formation. Developmental stages are as described by Kimmel et al. (Kimmel et al., 1995). robo2 (astray) homozygous mutant larvae (astray ${ }^{\text {ti272ziti272z }}$ ) were obtained from the Chien laboratory (University of Utah, Salt Lake City, UT, USA) (Fricke et al., 2001).

\section{Generation of transgenic fish lines}

The IslISS:Kaede reporter construct was generated by replacing the coding sequence of eGFP from the $\mathrm{Tg}$ (sensory:gfp) construct (Sagasti et al., 2005) with the coding sequence of Kaede (Ando et al., 2002). Isl1SS:Kaede germline transgenic fish were generated by co-injecting plasmid DNA and I-SceI meganuclease into one-cell stage embryos (Thermes et al., 2002). One stable transgenic line was recovered. Kaede expression is variegated within each batch of embryos, which is likely to be due to epigenetic silencing of UAS elements (Goll et al., 2009). Isl1SS:Kaede larvae with high Kaede expression levels were used for analysis.

The Trpalb:GFP reporter construct was generated by ET recombination of a bacterial artificial chromosome (BAC) (Zhang et al., 1998). BAC clone CHORI211-236I20 contains $115 \mathrm{~kb}$ upstream of the zebrafish Trpalb translation start site followed by a $52 \mathrm{~kb}$ region that encodes the extracellular domain of Trpalb. The eGFP gene and the kanamycin resistance gene were inserted at the trpalb translation start site, replacing the first two trpalb exons. The recombinant clone was validated by PCR, sequencing and transient expression assays. To generate a stable transgenic line, linearized BAC DNA was injected into one-cell stage zebrafish embryos followed by screening of adults for fluorescent progeny. One stable transgenic line was recovered. We and others (C. B. Chien, personal communication) have been unable to generate transgenic lines that allow the expression of full-length Robo2 under UAS control.

\section{Subtype-specific single trigeminal sensory neuron labeling}

To label single Isl1SS:Kaede trigeminal sensory neurons, $1 \mathrm{nl}$ of $10 \mathrm{pg} / \mathrm{nl}$ Isl1SS:Kaede plasmid DNA was injected into the yolk of one-cell stage embryos. Injected embryos were kept in the dark and screened at 2-3 days post-fertilization (dpf) for labeling of single trigeminal sensory neurons. Trigeminal sensory neurons were then photoconverted with a $405 \mathrm{~nm}$ confocal laser, as previously described (Caron et al., 2008). To label single
Trpalb:GFP trigeminal sensory neurons, $0.5 \mathrm{nl}$ of $45 \mathrm{pg} / \mathrm{nl}$ Trpalb:GFP BAC DNA was injected into a single cell of a four- to eight-cell stage embryo. Injected embryos were screened at 2-3 dpf. To label presynaptic puncta, Isl1SS:Gal4 was co-injected with the UAS-Syp:GFP-DSR plasmid, obtained from the Meyer laboratory (King's College London, London, UK) (Meyer and Smith, 2006).

\section{Image acquisition and processing}

All images were acquired using the FV1000 laser-scanning confocal imaging system (Olympus, Tokyo, Japan) on an upright microscope with a $20 \times$ XLUMPlanFl water-immersion objective. Larvae were anesthetized with $0.01 \%$ tricaine methanesulfonate (MS-222, Sigma) and transferred to a glass-bottomed Petri dish (P35G-0-14-C, MatTek). Molten 1.5\% lowmelt agarose (UltraPure LMP agarose, Invitrogen), kept on dry heat at $40^{\circ} \mathrm{C}$, was then added to the dish. Fish were arranged so that the surface to be imaged was facing the glass bottom. The dish was inverted for imaging (glass side up). For multi-time point experiments, larvae were released from the agarose after imaging with fine forceps and returned to a $28.5^{\circ} \mathrm{C}$ incubator for recovery.

Images were processed using FluoView (Olympus), ImageJ (NIH, http://rsbweb.nih.gov/ij/) and Photoshop (Adobe Systems, San Jose, CA, USA) software. Varicosities were counted manually. Branch length was measured using the NeuronJ plug-in in ImageJ. Hindbrain segments were delineated using the following criteria: segment 1 was defined as areas anterior to the anterior (utricular) otolith (AO); segments 2-4 were located between the $\mathrm{AO}$ and the posterior third of the posterior (saccular) otolith (PO); segments 5-7 were located between the posterior third of the PO and the posterior boundary of the first somite; segments 8-9, 10-11, 12-13 and 14-15 corresponded to the anterior and posterior halves of somites 2, 3, 4 and 5, respectively (Fig. 4A). To correlate segments with rhombomeres, hindbrain cranial motor nuclei were labeled using the Islet1:GFP transgenic line (Higashijima et al., 2000). Using cranial motor nuclei as markers, the positions of rhombomeres 2-8 were identified and mapped onto the segments as defined above (Ma et al., 2009; Mapp et al., 2011) (Fig. 4A, supplementary material Fig. S1). Varicosities were assigned to segments where they were physically located, whereas branch number and branch length were assigned to segments where branches originated.

\section{Statistical analysis}

One-way analysis of variance (ANOVA) with Newman-Keuls post test was used to compare total varicosity number, branch number and branch length between different genotypes and sensory neuron subtypes. Two-way ANOVA with Bonferroni post test was used to compare the morphological features of different genotypes or sensory neuron subtypes at a given anterior-posterior segment or time point. Statistical tests and $P$-values were calculated using Prism statistical software (GraphPad, La Jolla, CA, USA).

\section{Fluorescent in situ hybridization and immunohistochemistry} trpv1, trpalb and $p 2 \times 3 b$ ( $p 2 r \times 3 b-$ Zebrafish Information Network) DIGlabeled antisense RNA probes were synthesized as previously described (Caron et al., 2008). trkA, trkC1 and trkC2 (ntrk1, ntrk3a and ntrk3b, respectively - Zebrafish Information Network) probes were generated by 5'RACE (SMART RACE cDNA Amplification Kit, Clontech, Mountain View, CA, USA) using 3' primers based on Ensembl exon predictions. The cgrp (calca - Zebrafish Information Network) probe was generated by RTPCR with Superscript II reverse transcriptase (Invitrogen) using primers based on Ensembl exon predictions. Sequences are available from GenBank (trkA, JN837101; trkC1, JN837102; trkC2, JN837103; cgrp, JN837104), robo2 and Slit gene probes were obtained from the Chien laboratory (Hutson and Chien, 2002; Hutson et al., 2003; Lee et al., 2001).

Fluorescent in situ hybridization was performed using protocols described previously (Schoenebeck et al., 2007). In brief, embryos were hybridized with DIG-labeled RNA probes overnight at $68^{\circ} \mathrm{C}$ followed by stringent washes. Samples were incubated with anti-DIG POD-conjugated Fab fragments (Roche, 1:400) and mixed with Cy3-labeled tyramide (PerkinElmer, 1:25). GFP- or Kaede-labeled neurons were identified by incubation with rabbit anti-GFP or rabbit anti-Kaede antibody, respectively (MBL International, 1:1000). Trigeminal sensory neurons were identified with anti-HuC/D (Elavl3/4) antibody (Invitrogen, 1:1000). Fluorescent 
secondary antibodies coupled to Alexa dyes were used to detect primary antibodies (Invitrogen, 1:500). To distinguish GFP and Kaede in Trpa1b:GFP;Isl1SS:Kaede double transgenics, GFP was stained with a mouse anti-GFP antibody (Roche, 1:300) coupled to Alexa 647 (near infrared), whereas Kaede was stained with rabbit anti-Kaede coupled to Alexa 546 (red).

\section{RESULTS}

\section{Subtype-specific gene expression of trigeminal sensory neurons}

To explore how sensory neuron subtypes form distinct axonal projections, we aimed to identify genetically defined trigeminal sensory neuron subpopulations in larval zebrafish. We had previously found that Trpalb, the zebrafish homolog of the mammalian TRPA1 channel, is expressed in a subset of trigeminal sensory neurons and is required for sensitivity to several environmental and endogenous chemical irritants (Caron et al., 2008; Prober et al., 2008). To label this subpopulation of nociceptive neurons, we generated a Trpalb:GFP BAC transgenic line (Fig. 1A-C). GFP was observed in trigeminal sensory neurons and Rohon Beard sensory neurons (the spinal cord equivalents of the trigeminal sensory neurons), similar to endogenous trpalb expression (Prober et al., 2008). We also observed non-specific GFP expression in olfactory neurons, the retina and the tectum. To test whether GFP expression within the trigeminal ganglion is specific, we performed trpalb in situ hybridization in Trpalb:GFP transgenic larvae and found that GFP accurately marked trigeminal sensory neurons that expressed trpalb (supplementary material Fig. S2). Consistent with Trpalb being a subtype-specific marker, GFP expression was seen in a small subset of trigeminal sensory neurons $(10.52 \pm 0.51$ cells out of 60 neurons at $2 \mathrm{dpf})$.

To identify an additional trigeminal sensory neuron subtype, we generated a second reporter line, IsllSS:Kaede. This reporter line uses zebrafish islet 1 enhancer elements to drive gene expression in somatosensory neurons (Fig. 1A-C) (Higashijima et al., 2000; Sagasti et al., 2005). Kaede expression was seen in a subset of trigeminal sensory neurons ( $11.53 \pm 0.65$ cells/ganglion) that were largely distinct from Trpalb:GFP-expressing neurons $(12 \%$ overlap; $1.8 \pm 0.4$ double-positive neurons/ganglion; $n=238$ Trpalb: $\mathrm{FP}^{+}$neurons; Fig. 1C,D). Additionally, neither subpopulation overlapped with the larger trkA (nerve growth factor receptor)-expressing population (17.38 \pm 1.66 cells/ganglion) (Knaut et al., 2005; Liu and Ma, 2011; Martin et al., 1995) (Fig. 1D, supplementary material Fig. S3). These results indicate that Trpalb and Isl1SS label specific trigeminal sensory neuron subpopulations.

To further examine whether Trpalb and IsllSS subsets are distinct subtypes, we tested a panel of sensory neuron subtype markers by whole-mount in situ hybridization and antibody staining against GFP (in Trpalb:GFP) or Kaede (in Isl1SS:Kaede) (Fig. 1E, supplementary material Fig. S3). Trigeminal sensory neurons were identified based on their location, morphology and expression of the pan-neuronal marker $\mathrm{HuC} / \mathrm{D}$. $t r k C 1$, which encodes a receptor for Neurotrophin 3 (Martin et al., 1998; Williams et al., 2000), was preferentially expressed in the Trpalb subset (94\% of Trpalb ${ }^{+}$neurons) compared with Isl1SS (24\% of IsllSS neurons). A similar trend was observed with the trkC paralog trkC2. Several markers of nociceptive neurons were also differentially expressed: $p 2 \times 3 b$ (ATP receptor and marker for non-peptidergic nociceptors) was preferentially expressed in Trpalb neurons (100\%, versus $24 \%$ in Isl1SS), whereas calcitonin gene-related peptide (cgrp; a marker for peptidergic nociceptors) was preferentially expressed

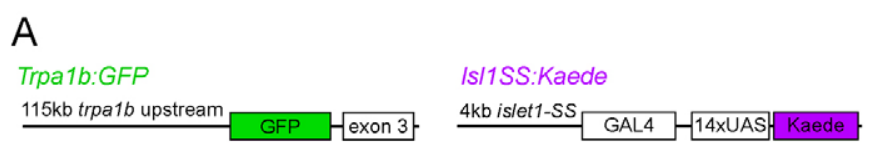

$\mathrm{B}$

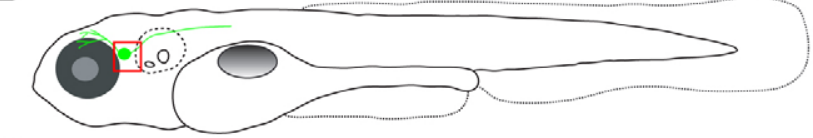

C

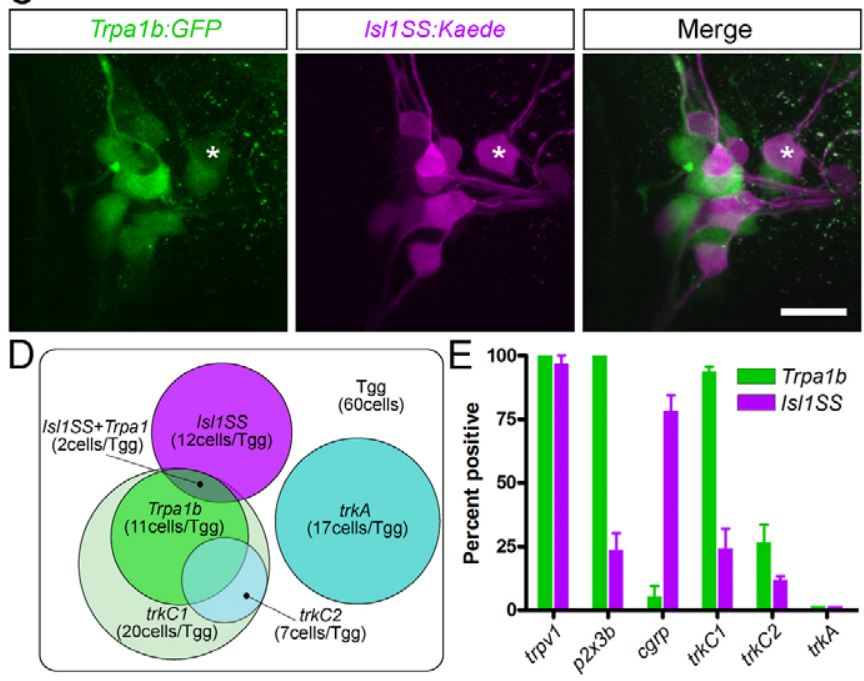

Fig. 1. Trigeminal subtype reporter lines and marker gene expression. (A) Transgenic constructs used to generate Trpa1b:GFP (left) and Is/1SS:Kaede (right) transgenic lines. (B) Illustration of a trigeminal sensory neuron (green cell abutting the eye) in a fish at 5 $\mathrm{dpf}$. The trigeminal ganglion (boxed area) is shown in C. (C) Trigeminal ganglion in a $5 \mathrm{dpf}$ Trpa1b:GFP;IS/1SS:Kaede double-transgenic larva. GFP-positive (green) and Kaede-positive (magenta) trigeminal sensory neurons are largely distinct. Asterisk indicates a double-labeled cell. (D) Summary Venn diagram of distinct trigeminal subtypes defined by neurotrophin receptors (trkA and trkC1) and transgenic reporters. The box represents the entire trigeminal ganglion (Tgg) and circles indicate populations of trigeminal sensory neurons labeled by the markers indicated. (E) Quantification of marker gene expression (\%) in each subset at $2 \mathrm{dpf}$. Error bars indicate s.e.m. Three to ten ganglia were counted for each marker. Scale bar: $20 \mu \mathrm{m}$.

in IsllSS neurons (78\%, versus 6\% in Trpalb neurons). These results establish that Trpalb and IsllSS neurons belong to different subtypes of trigeminal sensory neurons.

\section{Subtype-specific morphologies of trigeminal sensory neurons}

In addition to specific gene expression profiles, neuronal cell types are defined by morphological properties such as the position and branching pattern of neurites. These features also provide clues as to the connectivity patterns of the overall neural circuit (Masland, 2004). To test whether Trpalb and IsllSS neurons are morphologically distinct, we investigated the branching pattern and target specificity of their afferent axons by in vivo imaging. To obtain sparse labeling and allow unambiguous tracing and measurement of axon collaterals, we used DNA microinjection to generate mosaic transgenic fish with only one trigeminal sensory neuron labeled per ganglion (Fig. 2A-C). We found that Trpalb and IsllSS axons were distinct and stereotyped. The main axon 

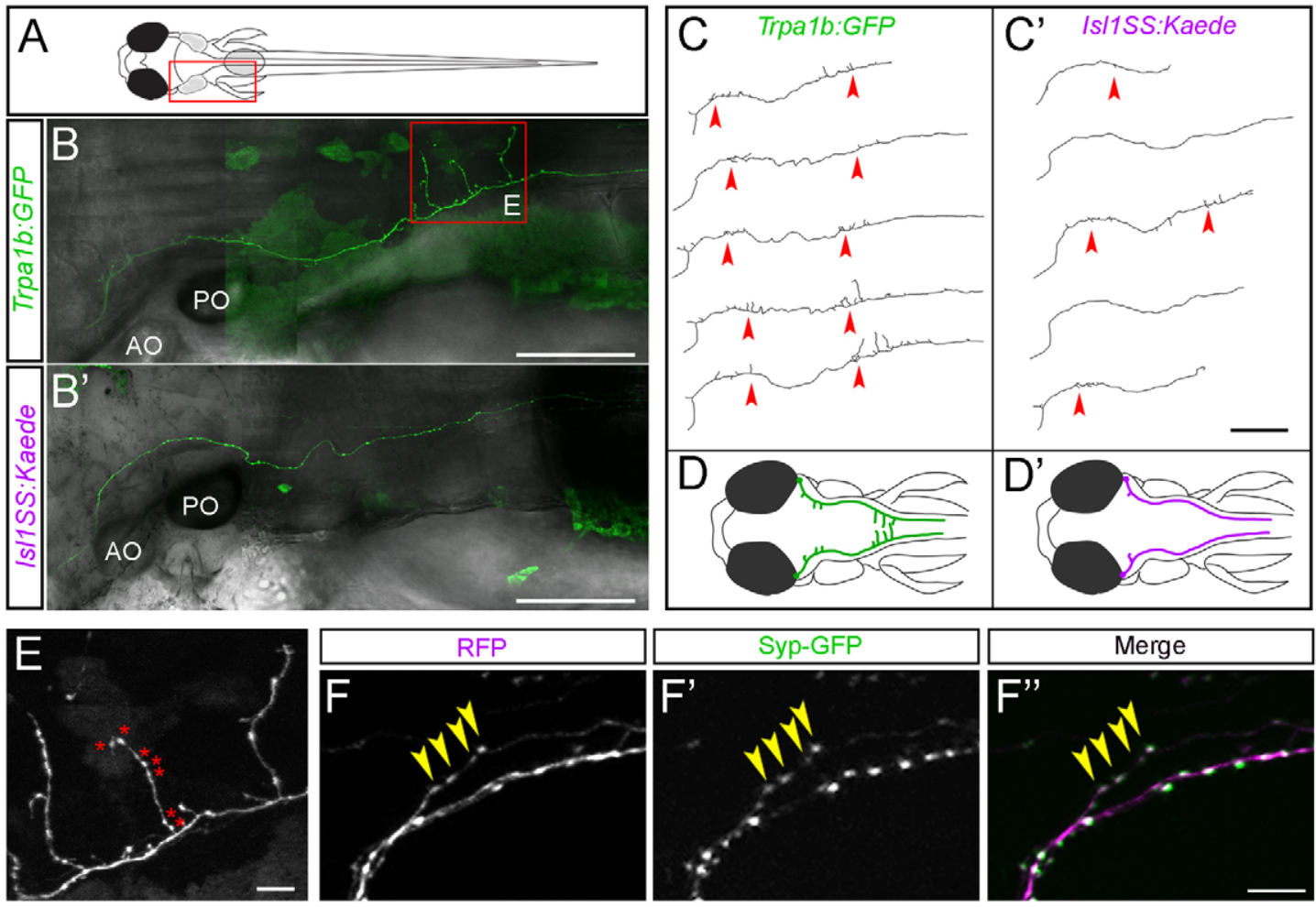

Fig. 2. Mosaic single-cell labeling of trigeminal sensory neuron subtypes. (A) Schematic of a 5-dpf larval zebrafish, viewed from the dorsal side. The boxed area corresponds to the imaged areas shown in $\mathbf{B}, \mathbf{B}^{\prime}$. (B, $\left.\mathbf{B}^{\prime}\right)$ In vivo imaging of single trigeminal sensory neurons. Fluorescent image is shown in green and bright-field image is superimposed in gray. Extensive branch growth is seen in the Trpa1b subset (B), but not the Is/1SS subset $\left(B^{\prime}\right)$. Boxed area corresponds to imaged area shown in $E$. $\left(\mathbf{C}, \mathbf{C}^{\prime}\right)$ Axon traces of multiple trigeminal sensory neurons are shown for both subtypes. Red arrowheads point to branches that form along the main axon tract. (D, $\left.\mathbf{D}^{\prime}\right)$ Illustrations of trigeminal afferents from Trpa $1 b$ (D) and Is/1SS (D') subtypes. (E) High-magnification image of boxed area in B. Trpa1b axon extends branches and forms numerous varicosities (asterisks). (F-F") Trigeminal axon labeled with cytoplasmic RFP (magenta, F) and Synaptophysin-GFP (Syp-GFP, green, F'), a fluorescent label for synaptic vesicles (with merge in $\mathrm{F}^{\prime \prime}$ ). The presence of varicosities reliably predicts the presence of Syp-GFP puncta (yellow arrowheads). AO, anterior otolith; PO, posterior otolith. Scale bars: $100 \mu \mathrm{m}$ in $\mathrm{B}-\mathrm{C}^{\prime} ; 10 \mu \mathrm{m}$ in $\mathrm{E}-\mathrm{F}^{\prime \prime}$.

shaft follows a similar trajectory in both subtypes. However, compared with Isl1SS neurons, Trpalb axons had more branches and increased total branch length (Fig. 2D, Fig. 3A,B). Trpalb axons also tended to extend further down the spinal cord than Isl1SS axons. In both subtypes, numerous varicosities were seen along the main axon shaft and on axon collaterals (Fig. 2E). Axonal varicosities, as seen by cytoplasmic fluorescent protein labeling, have previously been found to represent presynaptic puncta in many different zebrafish neuronal cell types (Appelbaum et al., 2010; Campbell et al., 2007; Meyer and Smith, 2006). To test whether this is also the case for trigeminal sensory neuron afferents, we co-expressed a red fluorescent protein (RFP) and Synaptophysin-GFP, a marker of presynaptic puncta, using the Isl1SS promoter (Meyer and Smith, 2006). We found that varicosities and GFP puncta were colocalized (Fig. 2F), and the number of varicosities and puncta were significantly correlated $\left(\mathrm{R}^{2}=0.74, \quad P<0.0001\right.$; see supplementary material Fig. S4). Therefore, we used varicosities as a reporter for presynaptic puncta. Comparison of the two trigeminal sensory neuron subtypes revealed that Trpalb axons had significantly more varicosities than Isl1SS axons (83.54 \pm 3.04 versus $55.84 \pm 3.36, P<0.001$; Fig. $3 \mathrm{C}$ ). Together, these results indicate that genetically defined trigeminal sensory neuron subtypes are morphologically distinct, both in branch morphology and the number of presynaptic puncta.

\section{Subtype-specific projection patterns of trigeminal sensory neurons}

The distinct morphologies of Trpalb and IsllSS neurons raised the possibility that these trigeminal subtypes have distinct projection patterns (Masland, 2004). To map the projections of individual afferents, we used mosaic transgenic labeling with combined fluorescent and bright-field imaging. The bright-field images, which show anatomical landmarks such as otoliths and somites, were used to delineate 15 anterior-posterior segments (Fig. 2A,B, Fig. 4A). These segments were reproducible between individual fish and correlated with rhombomeres 2-8 and anterior regions of the spinal cord (see Materials and methods). Using this anatomical map, the number of branches, the branch length and the number of presynaptic puncta (varicosities) of individual trigeminal sensory neurons were measured and compared between Trpalb and Isl1SS neurons.

The number of varicosities revealed subtype-specific patterns of innervation. Trpalb afferents showed two prominent peaks of high varicosity number, one in segments 3-4 and the other in segments 9-11 (Fig. 4B). By contrast, varicosities in the Isl1SS afferents showed a broader distribution, with only a minor peak in segment 5. The posterior Trpalb peak corresponded to the areas flanking the hindbrain-spinal cord junction, which is an important processing and relay area for trigeminal pain in mammals 

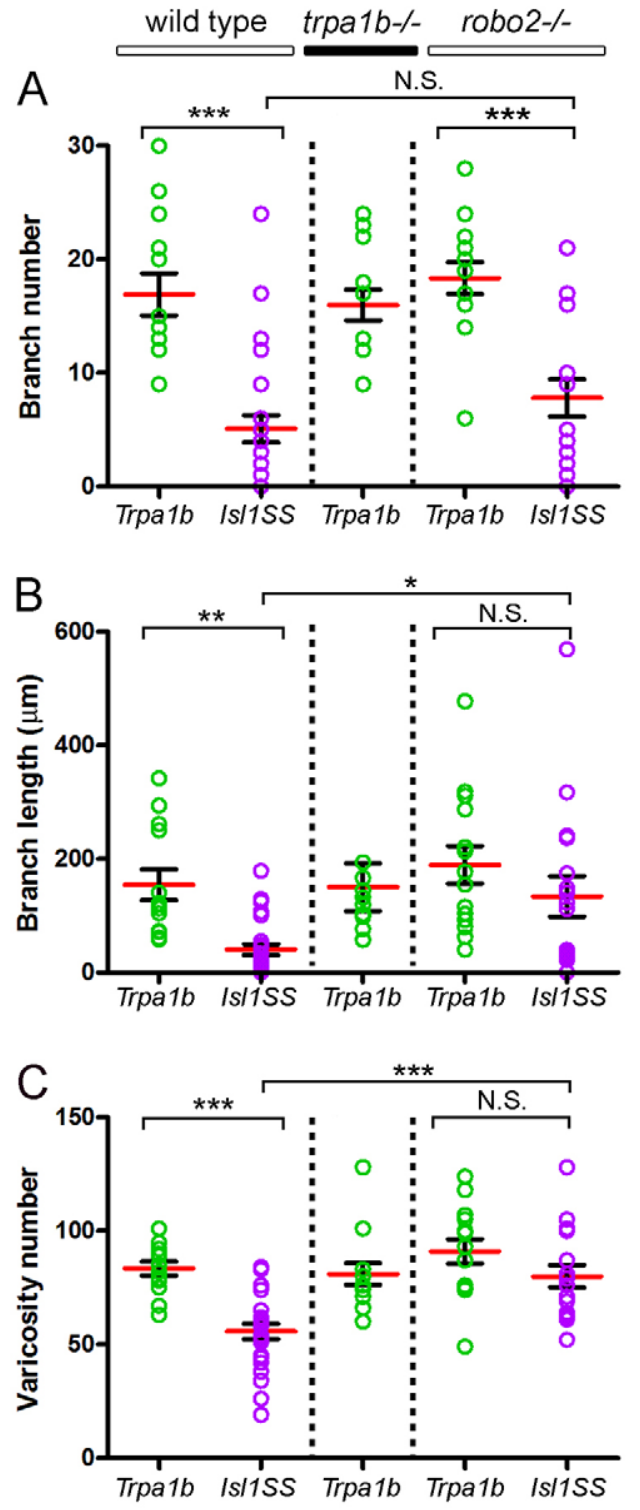

Fig. 3. Comparison of trigeminal sensory neuron subtype morphologies. Total branch number $(\mathbf{A})$, branch length $(\mathbf{B})$ and varicosity number $(\mathbf{C})$ compared between subtypes and genotypes. Red bar and black bracket are mean and s.e., respectively. Colored circles are values for individual trigeminal sensory neurons. Sample sizes are: 13, Trpa1b in wild type; 25, Is/1SS in wild type; $13, \operatorname{Trpa} 1 b$ in trpa $1 b^{-1-}$; 14, Trpa $1 b$ in robo2 ${ }^{--} ;$; 17 , Is/1SS in robo2 ${ }^{--}$. ${ }^{*}, P<0.05 ; * *, P<0.01$; $\star * *, P<0.001 ;$ N.S., no significant difference.

(Goadsby and Hoskin, 1997; Nash et al., 2009; Noma et al., 2008). Trpalb innervation in this region is consistent with its nociceptive function and suggests that the hindbrain-spinal cord junction might also be part of the nociceptive circuitry in zebrafish.

The distribution of branches also differed greatly between Trpalb and IsllSS (Fig. 4C,D). Trpalb afferents formed shorter branches in the anterior segments and longer branches in the posterior segments. Approximately one-quarter (3/13) of Trpalb trigeminal sensory neurons had posterior branches that reached the contralateral side. These contralaterally projecting branches are also seen in the posterior hindbrain of mammalian species and appear to be a feature of nociceptive fibers (Clarke and Bowsher, 1962;
A
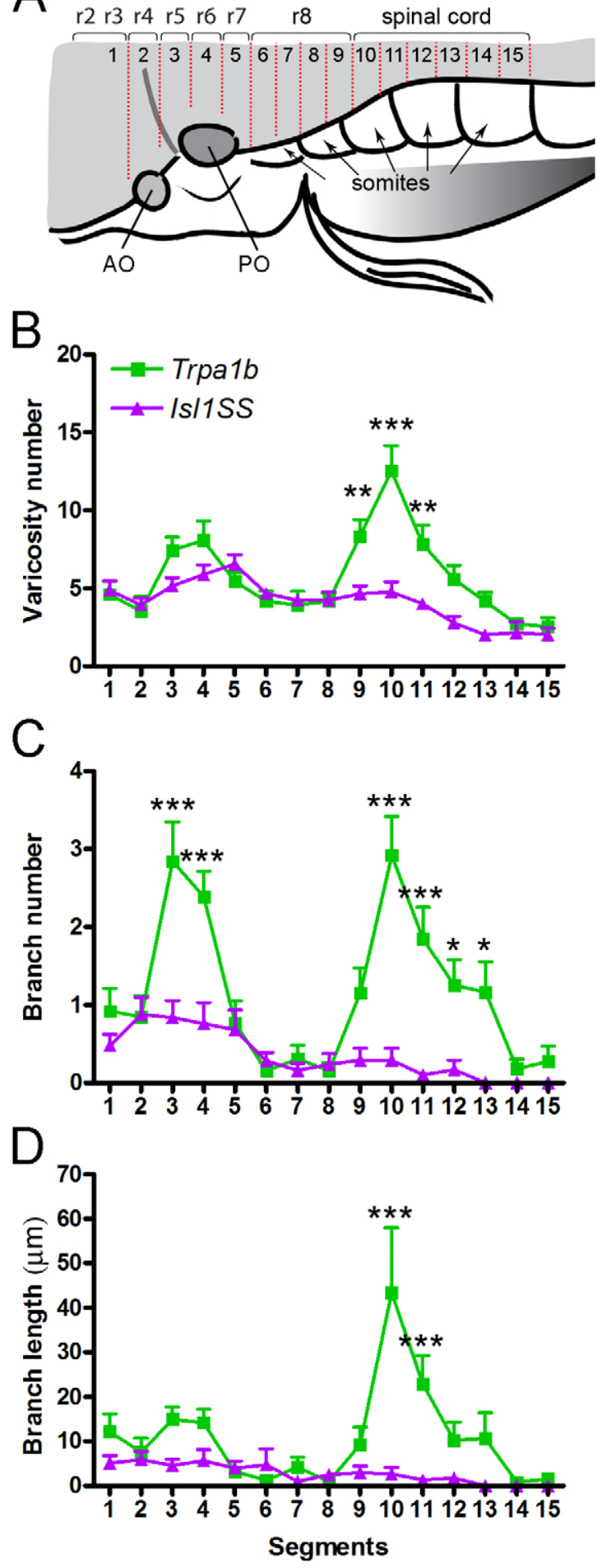

Fig. 4. Mapping subtype-specific axonal projections. (A) Branches and varicosities are mapped to defined segments in the zebrafish hindbrain and spinal cord along the anterior-posterior axis. Segments align with positions of rhombomeres ( $\mathrm{r} 1-\mathrm{r} 8)$ and somites.

(B-D) Distribution of varicosity number (B), branch number (C) and branch length (D) along segments 1-15. Similar trends are observed for a given subtype in all three parameters: Trpa $1 b$ neurons form a minor peak at segments 3-4 and a major peak at segments 9-11, whereas Is/1SS neurons do not form any pronounced peaks. Error bars indicate s.e.m. ${ }^{*}, P<0.05 ; * *, P<0.01 ; * * *, P<0.001$. AO, anterior otolith; $\mathrm{PO}$, posterior otolith. 


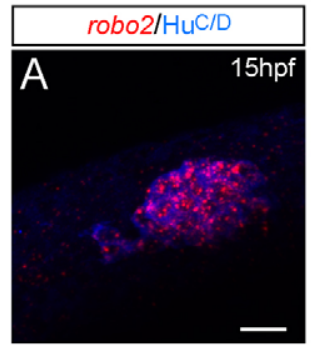

E

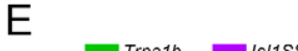

E Trpa1b $\square$ /s/1ss
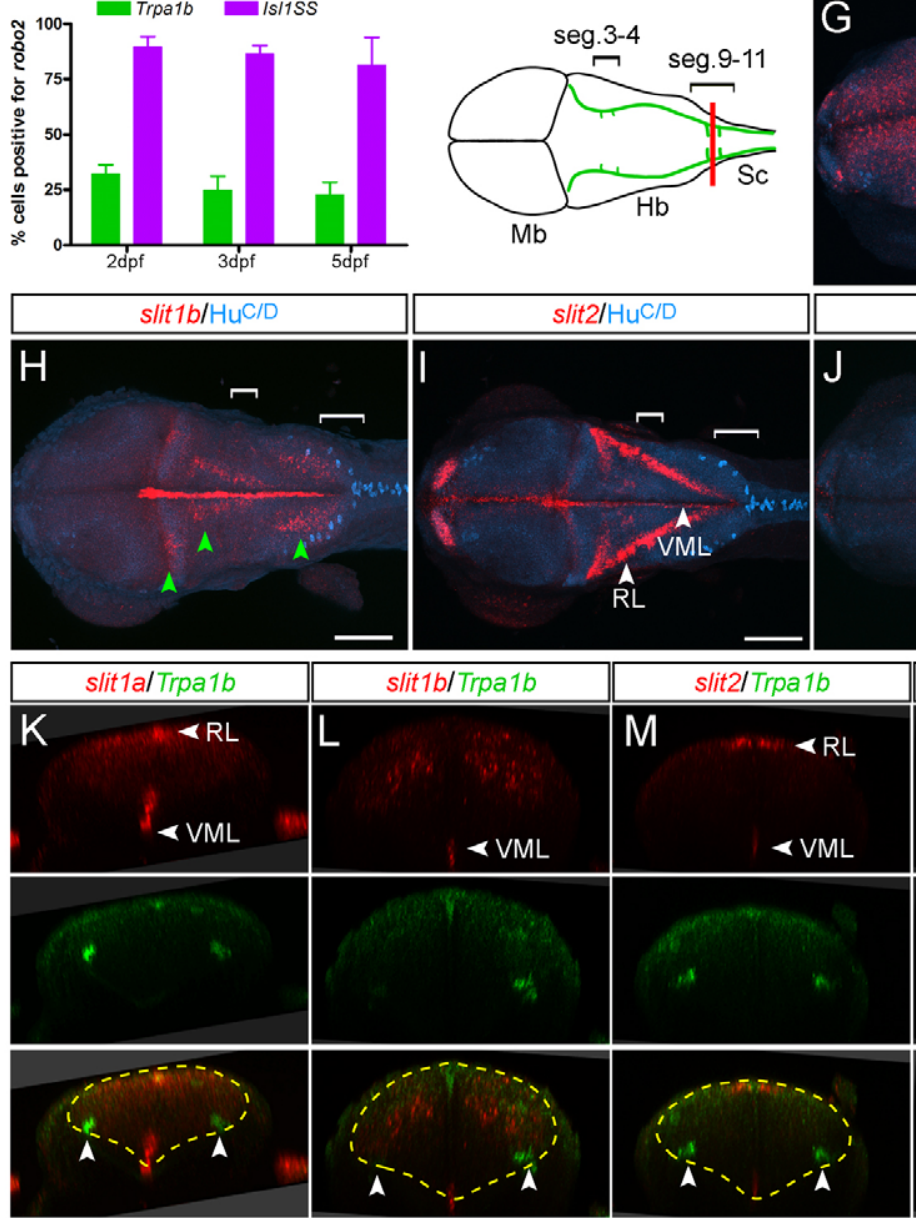

slit2ITrpa1b
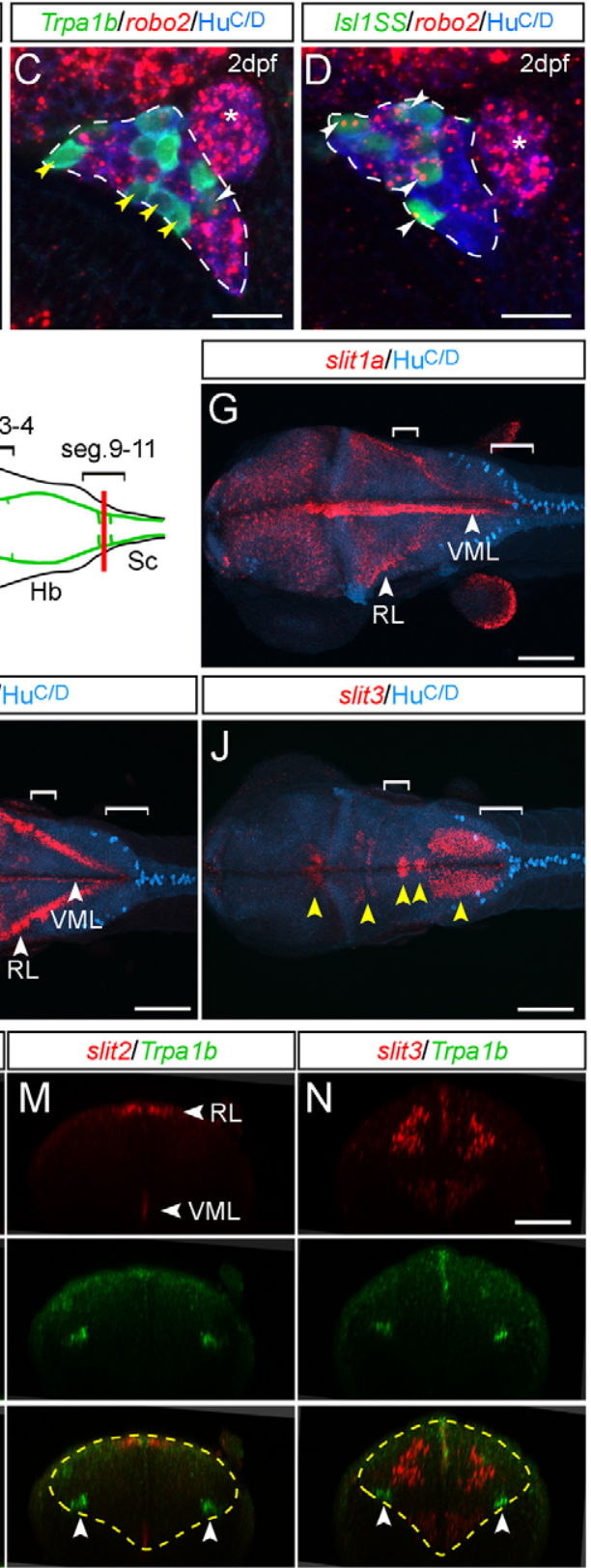

Fig. 5. Expression of robo2 and Slit genes. (A,B) Trigeminal ganglion (cluster of blue cells, HuC/D positive) expresses robo2 (red speckles) in most cells at early stages. (C,D) Starting at $2 \mathrm{dpf}$, robo 2 becomes differentially expressed in Tpra1b versus Is/1SS subtypes. The trigeminal ganglion is outlined (dashed lines); the anterior lateral line ganglion is located just posterior to it (asterisk) and is not labeled by either reporter line. (C) In the Trpa $1 b$ reporter line, most labeled trigeminal neurons (green cells) are negative for robo2 (yellow arrowheads), with a small proportion that are positive (white arrowhead). (D) In the Is/1SS reporter line, most labeled trigeminal neurons are positive for robo2 (white arrowheads).

(E) Quantification of robo2 expression in the two subtypes at different developmental stages. robo2 expression is consistently higher in the Is/1SS subtype from 2-5 dpf. Error bars indicate s.e.m. (F) Diagram of a 3 dpf zebrafish brain viewed from the dorsal side, corresponding to images shown in G-J. Green lines show the trajectory of the trigeminal afferent track. Brackets indicate segments 3-4 and 9-11. (G-J) Whole-mount in situ hybridization for Slit genes co-stained for a neuronal marker (HuC/D). Images are maximal projections of confocal z-stacks. $(G, I)$ slit1a and slit2 are strongly expressed in the ventral midline (VML) and rhombic lip $(\mathrm{RL})$. $(\mathrm{H})$ slit $1 \mathrm{~b}$ is expressed in the ventral midline as well as in several distinct nuclei in the hindbrain (green arrowheads). (J) slit3 is expressed in the cranial motor nuclei (yellow arrowheads). (K-N) Optical transverse sections at the hindbrain-spinal cord boundary (red line in F) of 3-dpf Trpa1b:GFP larvae stained for Slit genes (red) and Trpa 1b:GFP (green). slit1a is also diffusely expressed in the caudal hindbrain (K). Yellow dashed lines mark the outlines of the hindbrain and arrowheads indicate trigeminal axons. Mb, midbrain; $\mathrm{Hb}$, hindbrain; SC, spinal cord. Scale bars: $20 \mu \mathrm{m}$ in A-D; $100 \mu \mathrm{m}$ in G-J; $50 \mu \mathrm{m}$ in $\mathrm{K}-\mathrm{N}$.
Sugimoto et al., 1997a; Sugimoto et al., 1997b). By contrast, Isl1SS afferents had only a few short branches in the anterior segments and did not have contralaterally projecting afferents $(0 / 24)$. These results indicate that Trpalb and Isl1SS subpopulations have distinct and stereotypic axonal projections and branch growth patterns.

\section{Expression of robo2 and Slit genes marks trigeminal sensory neurons and afferent target fields}

What are the molecular mechanisms that establish subtype-specific afferent projections? The differential expression of trpalb itself does not appear to be involved because Trpalb neurons in trpalb mutants had normal axonal morphology (Fig. 3). We hypothesized that there might be subtype-specific growth-promoting or inhibitory cues that regulate this process and searched for signaling molecules that were differentially expressed in the two trigeminal subtypes. The axon guidance receptor Robo 2 is expressed in the rodent and zebrafish trigeminal ganglion, but it has been unclear whether it is expressed in all or a subset of sensory neurons and whether it exerts positive or negative effects on axon morphogenesis (Ma and Tessier-Lavigne, 2007; Ozdinler and Erzurumlu, 2002; Yeo et al., 2004). We therefore examined the expression of robo2 in more detail by fluorescent in situ hybridization. We found that robo2 was dynamically expressed in trigeminal sensory neurons during development. After initially broad expression (Fig. 5A,B), robo2 expression became restricted to a subset of trigeminal sensory neurons (Fig. 5C-E). Strikingly, more than $80 \%$ of IsllSS neurons and less than $30 \%$ of Trpalb neurons expressed robo 2 at 2-5 dpf. These results raised the possibility that Robo 2 expression might account for some of the differences in the morphology of IsllSS and Trpalb afferents.

To determine where Robo signaling might be activated, we examined the expression of Slits, the secreted ligands for Robo2. In the hindbrain, all four zebrafish Slit genes were expressed 

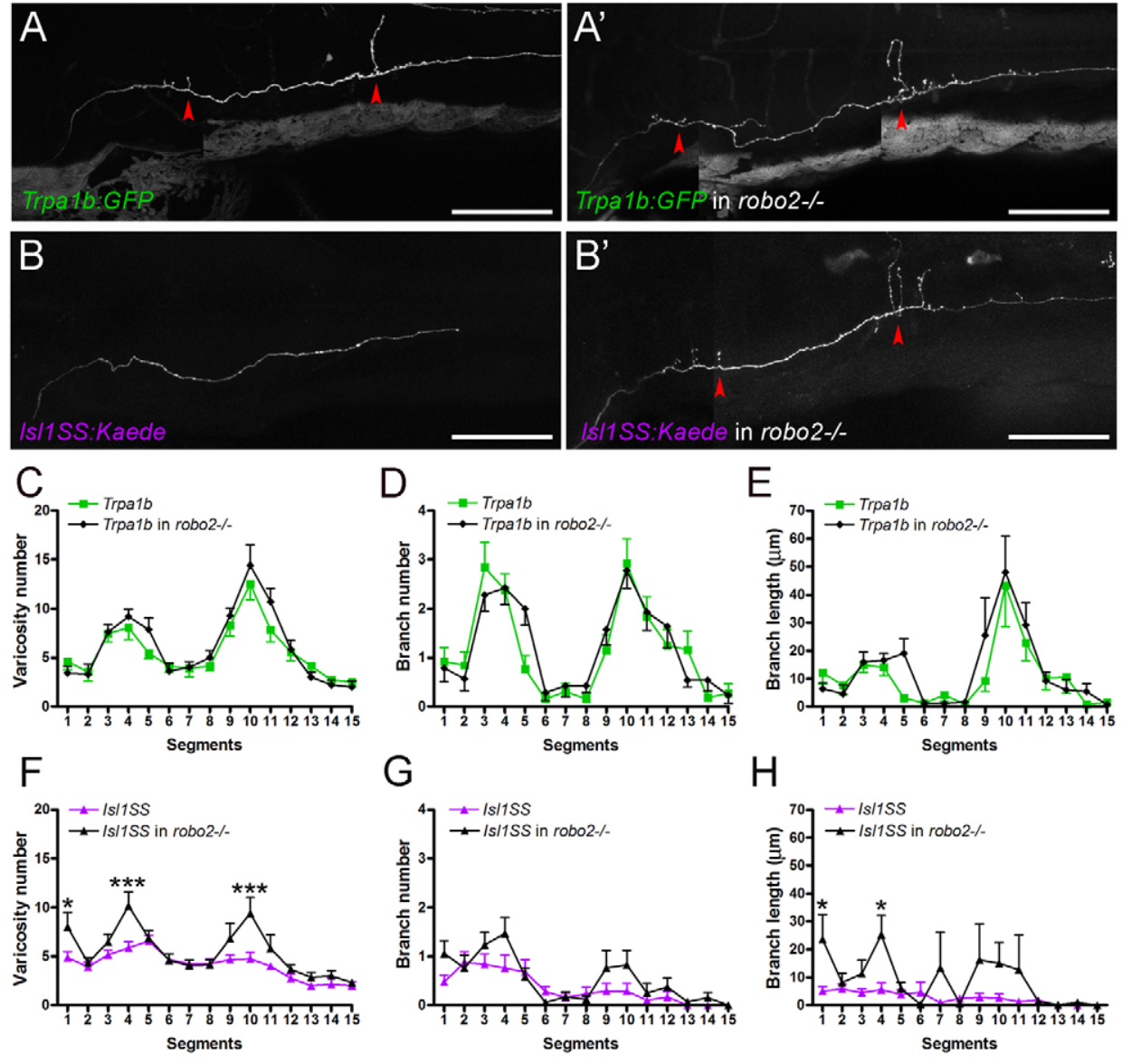

G

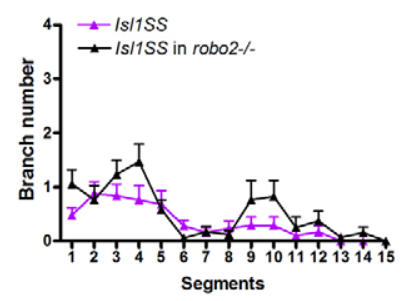

E

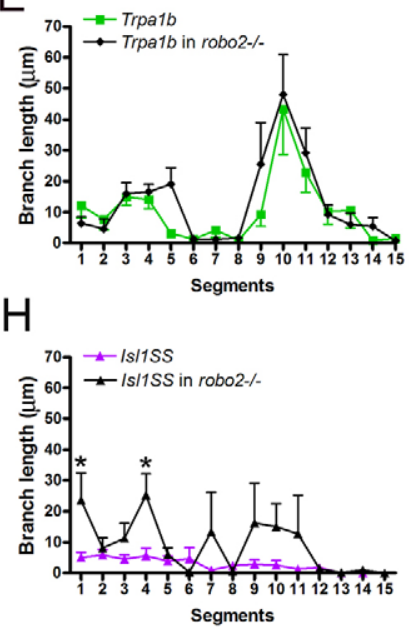

Fig. 6. Robo2 regulates subtypespecific projection patterns.

$\left(\mathbf{A}-\mathbf{B}^{\prime}\right)$ Trigeminal axonal morphology in wild-type and $\mathrm{robo}^{-/-}$zebrafish. Red arrowheads point to branches. Trpa $1 \mathrm{~b}$ axonal morphology in $\mathrm{robo}^{-/-}$is indistinguishable from that of wild type $\left(A, A^{\prime}\right)$. By contrast, there is a noticeable increase in branch length and varicosity number in the Is/1SS subtype in robo2 ${ }^{-1-}$ $\left(B, B^{\prime}\right)$. (C-E) Comparison of Trpa $1 b$ morphology in wild type (green) versus robo2 $^{-1-}$ (black). No differences were seen in varicosity number (C), branch number (D) or branch length (E).

(F-H) Comparison of Is/1SS morphology in wild type (magenta) versus robo $2^{-/-}$ (black). Significant differences were seen in varicosity number $(\mathrm{F})$ and branch length $(H)$, but not branch number $(G)$. Error bars indicate s.e.m. ${ }^{*}, P<0.05 ; * * *$, $P<0.001$. Scale bars: $100 \mu \mathrm{m}$.
(Fig. 5F-N). slit1a and slit2 were highly expressed in the ventral midline and the rhombic lip, relatively distant from the trigeminal target field. By contrast, slit $1 b$ and slit3 were expressed in discrete domains along the anterior-posterior axis, immediately dorsal-medial to the trigeminal afferent track. Slit expression partly overlapped with the anterior and posterior Trpalb peaks, but expression of individual Slit genes did not define its boundaries (Fig. 5F-J, brackets). These results reveal that robo2 expression is enriched in IsllSS neurons and that multiple Slit genes are expressed in the vicinity of the trigeminal sensory neuron afferents.

\section{robo2 regulates subtype-specific projection patterns}

Robo2 enrichment in Isl1SS neurons suggests that Robo/Slit signaling might regulate the afferent projection pattern in the Isl1SS subtype. To test this, we examined the axonal morphology of Trpalb and IsllSS subtypes in robo2 (astray) loss-of-function mutants (Fricke et al., 2001; Hutson and Chien, 2002). Strikingly, Isl1SS afferents were partially transformed to Trpalb-like morphology in robo2 mutants (Fig. 6A,B). In either subtype, there were no changes in branch number in robo $^{-/-}$relative to wild-type controls (Fig. 3A). However, Isl $1 S S$ sensory axons had significantly increased branch length and varicosity number in robo 2 mutants (Fig. 3B,C). By contrast, Trpalb neurons displayed no differences between mutants and controls. Notably, the subtype-specific differences in branch length and varicosity number between IsllSS and Trpalb axons were abolished in robo $2^{-/}$larvae. These results suggest that robo 2 acts to regulate subtype-specific branch length and presynaptic differentiation with no effect on branch formation.
To test whether Robo2 also determines the localization of varicosities and branches, we examined their anterior-posterior distribution in robo2 mutants (Fig. 6). The projection patterns for Trpalb neurons were not affected by loss of Robo2 (Fig. 6C). By contrast, Isl1SS neurons had increased varicosities in both anterior (segments 3-4) and posterior (segments 9-11) segments in robo2 mutants, compared with wild-type controls (Fig. 6F). Thus, Isl1SS and Trpalb neurons acquired very similar varicosity distributions. Segment-specific changes were also observed for branch number and branch length, but the changes were less pronounced than those observed for varicosities (Fig. 6D,E,G,H). These results indicate that signaling through robo 2 is a key regulator of the regionspecific morphology of a subset of trigeminal sensory neurons.

\section{Morphogenesis of subtype-specific branch growth and synaptogenesis}

Subtype-specific differences in branch morphology and synapse number may arise from (1) selective addition and growth in Trpalb afferents or (2) equal growth in both subpopulations followed by selective pruning in Isl1SS afferents. To distinguish between these possibilities, we analyzed the dynamics of branch growth and varicosity formation at the hindbrain-spinal cord junction by in vivo imaging (segments 9-11, Fig. 7A). At $2 \mathrm{dpf}$, Trpalb and Isl1SS neurons showed very similar morphology, with short branches and comparable varicosity number (Fig. 7B-E). Morphological changes arose between 2 and 3 dpf, when Trpalb neurons increased branch number, branch length and varicosity number. Branch growth slowed down over the next few days (4-5 dpf), while varicosity number steadily increased. IsllSS neurons, by contrast, had only modest increases in varicosity number and no changes in branch number and 

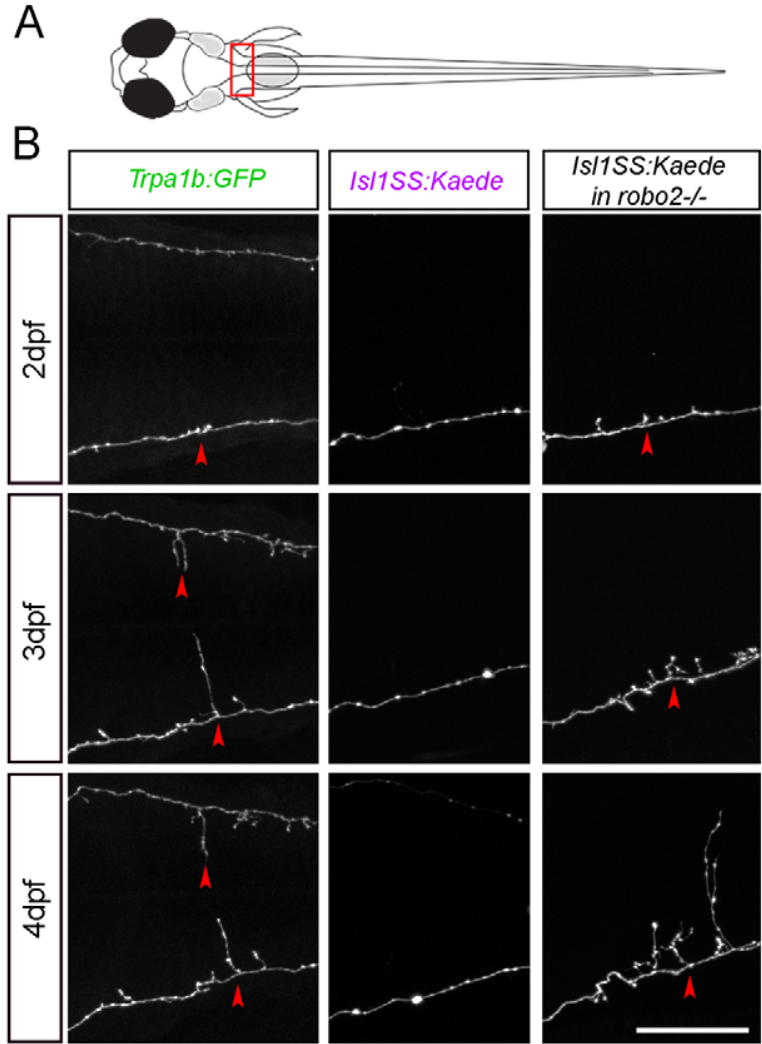

C
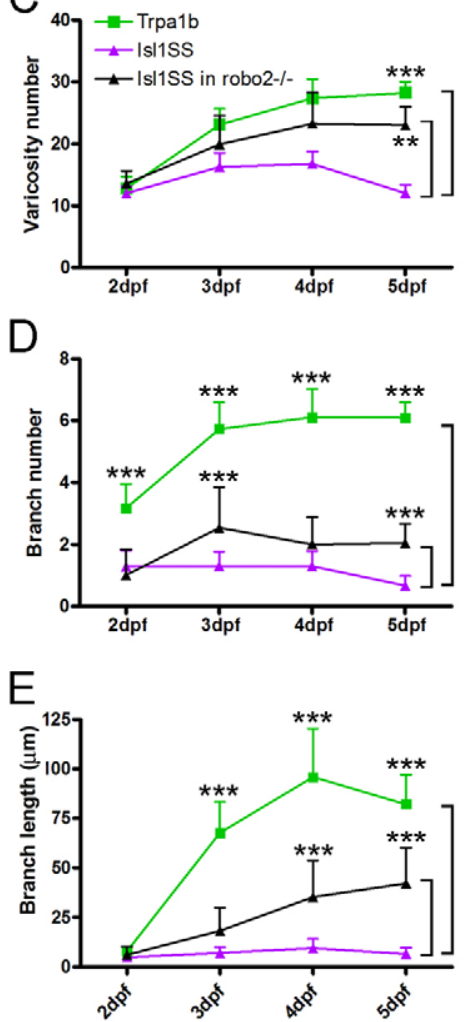

Fig. 7. Morphogenesis of subtypespecific branch growth and synaptogenesis. (A) Multi-time point imaging was performed in segments 911 (boxed area), where the innervation pattern differs most between Trpa $1 b$ and Is/1SS neurons. (B) Fluorescent images of individual trigeminal sensory neuron afferents imaged from 2 to $4 \mathrm{dpf}$. Representative examples are shown for Trpa $1 b$ (left column), Is/1SS (middle) and Is/1SS in robo2 $2^{-1-}$ (right) zebrafish. Red arrowheads point to branches.

(C-E) Afferent morphology is compared between genotypes (wild type versus robo $^{-1-}$ in the Is/1SS subtype) and subtypes (Trpa1 $b$ versus Is/1SS). Error bars indicate s.e.m. **, $P<0.01 ; * * *$,

$P<0.001$. Scale bar: $50 \mu \mathrm{m}$. branch length. These results reveal that the morphological divergence of trigeminal subtypes depends on selective growth and synaptogenesis rather than selective pruning.

To determine how Robo 2 affects axon morphogenesis, we examined the growth of IsllSS axons in robo2 mutants. Loss of robo2 did not affect axonal morphogenesis in early development (2 dpf), despite the early expression of robo2 in wild type (Fig. 7B, right column). As the afferent axons matured, robo $2^{-1-}$ IsllSS axons began to form more varicosities and branches, whereas their wildtype counterparts remained largely unchanged (Fig. 7C-E). These results reveal that Isl1SS axons have an intrinsic capacity for growth that is suppressed by Robo2.

\section{DISCUSSION}

The hindbrain is the first relay and processing station of somatosensory neural circuits. Sensory afferents carrying diverse sensory modalities, such as touch, chemicals and temperature, project to specific regions in the hindbrain and spinal cord. Our study defines a mechanism by which two trigeminal sensory neuron subtypes acquire distinct afferent morphologies and axon projections: subtype-specific expression of Robo2 inhibits branch growth and presynaptic terminal formation (Fig. 8).

\section{Regulation of axonal morphology in somatosensory subtypes}

There is a growing understanding of the molecular and physiological properties of different trigeminal sensory neuron subtypes and their afferent targets (Liu and Ma, 2011; Perl, 2007). However, it has been unclear whether systematic differences exist in the afferent branching patterns and synaptic densities between defined trigeminal sensory neuron subtypes (Hayashi, 1985a; Hayashi, 1985b; Jacquin et al., 1986; Light and Perl, 1979). Our results reveal that subclasses of trigeminal sensory neurons have overall similar axon trajectories but display very different afferent branching morphologies. Differences in Robo/Slit signaling play a major role in ensuring subtype-specific projections. Most notably, Robo2 acts to dampen synaptogenesis and branch growth but not branch number in the IsllSS subtype.

In the hindbrain, Slit proteins are expressed in the floor plate, rhombic lip and in several hindbrain nuclei (Hammond et al., 2005; Marillat et al., 2002; Yuan et al., 1999). The expression of Slit genes might prevent trigeminal afferents from forming inappropriate contacts with Slit-expressing cells. We found slit3 expression in the cranial motor nuclei, similar to mammalian Slit2/3 expression (Geisen et al., 2008). Several cranial motor nuclei (V, VII and XII) are involved in the nociceptive reflex triggered by strong trigeminal stimulation, but they are not directly connected to trigeminal sensory neurons (Dong et al., 2011). Given the close proximity of cranial nuclei and trigeminal afferents, Robo2/Slit3 signaling might be required to prevent erroneous innervations.

In addition to cell type-specific inhibition mediated by Robo2 signaling, growth-promoting signals may also play a role in establishing sensory subcircuits. In the absence of Robo2 inhibition, we found that IsllSS neurons are also able to increase growth and synaptogenesis in the same Tpralb peak segments. This suggests that both subtypes can respond to a putative growthpromoting signal that might be localized to these segments. Previous studies have identified potential candidates for such a signal. For example, expression studies in other model systems suggest that multiple axon guidance pathways are active in trigeminal sensory neurons, including Neurotrophin/Trk, Netrin/Unc5 and Semaphorin/Neuropilin (Erzurumlu et al., 2010; Masuda et al., 2008). Other Robo family members (Robol and Robo3) might also play a role (Ma and Tessier-Lavigne, 2007). It is a challenge for the future to identify potential growth-promoting signals and investigate their interactions with the Robo2 pathway. 


\section{Late development}

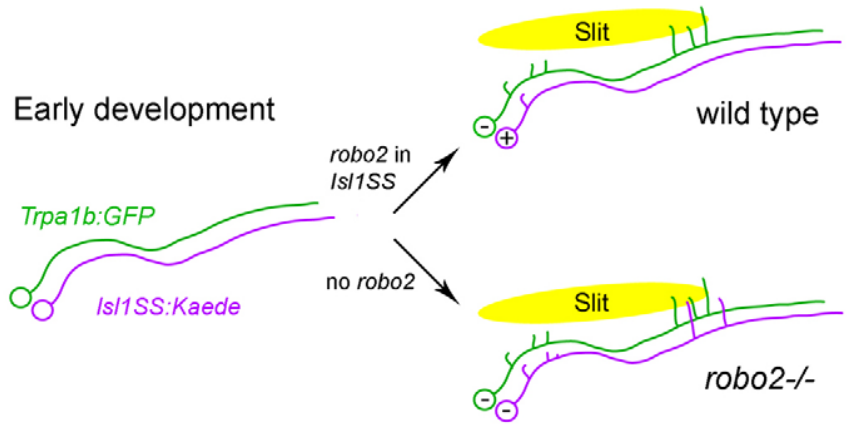

Fig. 8. Robo2-dependent morphogenesis in trigeminal sensory neurons. Trigeminal sensory neurons from Is/1SS (magenta) and Trpa1b (green) subtypes have similar morphology during early development. In wild-type fish, developmental maturation leads to preferential expression of robo2 in the Is/1SS subtype (marked by ' + ') but not in the Trpa $1 b$ subtype (marked by ' - '). Robo2, which is likely to be activated by secreted Slit proteins in the hindbrain (yellow oval), inhibits branch growth and synaptogenesis specifically in the Is/1SS subtype. In robo2 $2^{-/}$ fish, both subtypes lack Robo2. The Is/1SS subtype, now relieved of Robo2 inhibition, extends axon branches and forms synapses in the same areas as in the Trpa $1 b$ subtype.

\section{Functional implications of subtype-specific projection patterns}

Somatosensory afferents innervate selective hindbrain and spinal cord regions in a cell type-specific manner and thereby activate divergent downstream targets to initiate distinct behavioral responses (Braz et al., 2005; Brodal, 2010). We observed two regions, one anterior and one posterior, where Trpalb and Isl ISS neurons showed different innervation density (as measured by varicosity number). The anterior region (segments 3-4) corresponds to rhombomeres 56 , which contain reticulospinal neurons (the Mauthner array neurons) important for the trigeminal-mediated fast escape response in zebrafish (Caron et al., 2008; Douglass et al., 2008; Kohashi and Oda, 2008; Liu and Fetcho, 1999; Sagasti et al., 2005). The observed high (in Trpalb) and low (in IsllSS) innervation density in this region suggests that there might be subtype-specific patterns of Mauthner array activation and initiation of the fast escape response.

The posterior region (segments 9-11) corresponds to the caudalis subnuclei of the $\mathrm{SpV}$, which is known to be crucial for trigeminal sensory neuron-mediated pain (e.g. toothache, headache, migraine) (Sessle, 2000). Selective innervation by nociceptive Trpalb afferents suggests that there might be functional similarity between fish and mammals in this anatomical region. The identities and function of Trpalb target cells are not yet known, but this region has recently been proposed to contain specialized cells that can initiate persistent swimming (Kyriakatos et al., 2011). This would be consistent with the observation that Trpalb activation increases overall motor activity (Prober et al., 2008) and raises the possibility that IslISS and Trpalb subtypes have distinct abilities to trigger persistent swimming.

\section{Acknowledgements}

We dedicate this study to C. B. Chien and thank him for the robo2 and Slit gene probes, robo2 (astray) mutant fish and for many thoughtful suggestions. We thank D. Yelon for the CHORI211-236I20 BAC clone; M. Meyer for the Syp:GFP-DSR plasmid; S. Zimmerman, J. Miller and K. Hurley for fish care; P. Huang, F. Imam, J. Kay, F. Merkle and X. Wang for critical comments on the manuscript; and members of the Schier laboratory for discussion.

\section{Funding}

This work was supported by a National Research Service Award (NRSA) postdoctoral fellowship from the National Institutes of Health [F32 NS057870 to Y.A.P.]; and research grants from the National Institutes of Health [R01 HD067140 and R01 NS049319 to A.F.S.]. Deposited in PMC for release after 12 months.

Competing interests statement

The authors declare no competing financial interests.

Supplementary material

Supplementary material available online at

http://dev.biologists.org/lookup/suppl/doi:10.1242/dev.076588/-/DC1

\section{References}

Ando, R., Hama, H., Yamamoto-Hino, M., Mizuno, H. and Miyawaki, A. (2002). An optical marker based on the UV-induced green-to-red photoconversion of a fluorescent protein. Proc. Natl. Acad. Sci. USA 99, 12651-12656.

Appelbaum, L., Wang, G., Yokogawa, T., Skariah, G. M., Smith, S. J., Mourrain, P. and Mignot, E. (2010). Circadian and homeostatic regulation of structural synaptic plasticity in hypocretin neurons. Neuron 68, 87-98.

Basbaum, A. I., Bautista, D. M., Scherrer, G. and Julius, D. (2009). Cellular and molecular mechanisms of pain. Cell 139, 267-284.

Braz, J. M., Nassar, M. A., Wood, J. N. and Basbaum, A. I. (2005). Parallel 'pain' pathways arise from subpopulations of primary afferent nociceptor. Neuron $\mathbf{4 7}$, 787-793.

Brodal, P. (2010). The Central Nervous System: Structure and Function. Oxford: Oxford University Press.

Campbell, D. S., Stringham, S. A., Timm, A., Xiao, T., Law, M.-Y., Baier, H., Nonet, M. L. and Chien, C.-B. (2007). Slit1a inhibits retinal ganglion cell arborization and synaptogenesis via Robo2-dependent and -independent pathways. Neuron 55, 231-245.

Caron, S. J., Prober, D., Choy, M. and Schier, A. F. (2008). In vivo birthdating by BAPTISM reveals that trigeminal sensory neuron diversity depends on early neurogenesis. Development 135, 3259-3269.

Caterina, M. J., Schumacher, M. A., Tominaga, M., Rosen, T. A., Levine, J. D. and Julius, D. (1997). The capsaicin receptor: a heat-activated ion channel in the pain pathway. Nature 389, 816-824.

Cavanaugh, D. J., Chesler, A. T., Braz, J. M., Shah, N. M., Julius, D. and Basbaum, A. I. (2011). Restriction of transient receptor potential vanilloid-1 to the peptidergic subset of primary afferent neurons follows its developmental downregulation in nonpeptidergic neurons. J. Neurosci. 31, 10119-10127.

Chen, C. C., Akopian, A. N., Sivilotti, L., Colquhoun, D., Burnstock, G. and Wood, J. N. (1995). A P2X purinoceptor expressed by a subset of sensory neurons. Nature 377, 428-431.

Cho, J. H., Lepine, M., Andrews, W., Parnavelas, J. and Cloutier, J. F. (2007). Requirement for Slit-1 and Robo-2 in zonal segregation of olfactory sensory neuron axons in the main olfactory bulb. J. Neurosci. 27, 9094-9104.

Cho, J. H., Prince, J. E., Cutforth, T. and Cloutier, J. F. (2011). The pattern of glomerular map formation defines responsiveness to aversive odorants in mice. J. Neurosci. 31, 7920-7926.

Clarke, W. B. and Bowsher, D. (1962). Terminal distribution of primary afferent trigeminal fibers in the rat. Exp. Neurol. 6, 372-383.

Dhaka, A., Earley, T. J., Watson, J. and Patapoutian, A. (2008). Visualizing cold spots: TRPM8-expressing sensory neurons and their projections. J. Neurosci. 28, 566-575.

Dickson, B. J. and Gilestro, G. F. (2006). Regulation of commissural axon pathfinding by slit and its Robo receptors. Annu. Rev. Cell Dev. Biol. 22, 651-675.

Dong, Y., Li, J., Zhang, F. and Li, Y. (2011). Nociceptive afferents to the premotor neurons that send axons simultaneously to the facial and hypoglossal motoneurons by means of axon collaterals. PLOS ONE 6, e25615.

Douglass, A. D., Kraves, S., Deisseroth, K., Schier, A. F. and Engert, F. (2008). Escape behavior elicited by single, channelrhodopsin-2-evoked spikes in zebrafish somatosensory neurons. Curr. Biol. 18, 1133-1137.

Erzurumlu, R. S., Chen, Z.-F. and Jacquin, M. F. (2006). Molecular determinants of the face map development in the trigeminal brainstem. Anat. Rec. A Discov. Mol. Cell. Evol. Biol. 288, 121-134.

Erzurumlu, R. S., Murakami, Y. and Rijli, F. M. (2010). Mapping the face in the somatosensory brainstem. Nat. Rev. Neurosci. 11, 252-263.

Fricke, C., Lee, J. S., Geiger-Rudolph, S., Bonhoeffer, F. and Chien, C. B. (2001). astray, a zebrafish roundabout homolog required for retinal axon guidance. Science 292, 507-510.

Geisen, M. J., Di Meglio, T., Pasqualetti, M., Ducret, S., Brunet, J. F., Chedotal, A. and Rijli, F. M. (2008). Hox paralog group 2 genes control the migration of mouse pontine neurons through slit-robo signaling. PLoS Biol. 6, e142.

Goadsby, P. J. and Hoskin, K. L. (1997). The distribution of trigeminovascular afferents in the nonhuman primate brain Macaca nemestrina: a c-fos immunocytochemical study. J. Anat. 190, 367-375. 
Goll, M. G., Anderson, R., Stainier, D. Y., Spradling, A. C. and Halpern, M. E. (2009). Transcriptional silencing and reactivation in transgenic zebrafish. Genetics $182,747-755$

Hammond, R., Vivancos, V., Naeem, A., Chilton, J., Mambitisaeva, E., Andrews, W., Sundaresan, V. and Guthrie, S. (2005). Slit-mediated repulsion is a key regulator of motor axon pathfinding in the hindbrain. Development 132, 4483-4495.

Hayashi, H. (1985a). Morphology of central terminations of intra-axonally stained, large, myelinated primary afferent fibers from facial skin in the rat. J. Comp. Neurol. 237, 195-215.

Hayashi, H. (1985b). Morphology of terminations of small and large myelinated trigeminal primary afferent fibers in the cat. J. Comp. Neurol. 240, 71-89.

Higashijima, S., Hotta, Y. and Okamoto, H. (2000). Visualization of cranial motor neurons in live transgenic zebrafish expressing green fluorescent protein under the control of the islet-1 promoter/enhancer. J. Neurosci. 20, 206-218.

Hutson, L. D. and Chien, C. B. (2002). Pathfinding and error correction by retina axons: the role of astray/robo2. Neuron 33, 205-217.

Hutson, L. D., Jurynec, M. J., Yeo, S. Y., Okamoto, H. and Chien, C. B. (2003) Two divergent slit1 genes in zebrafish. Dev. Dyn. 228, 358-369.

Jacquin, M. F., Renehan, W. E., Mooney, R. D. and Rhoades, R. W. (1986) Structure-function relationships in rat medullary and cervical dorsal horns. I. Trigeminal primary afferents. J. Neurophysiol. 55, 1153-1186

Kay, J. N., De la Huerta, I., Kim, I. J., Zhang, Y., Yamagata, M., Chu, M. W., Meister, M. and Sanes, J. R. (2011). Retinal ganglion cells with distinct directional preferences differ in molecular identity, structure, and central projections. J. Neurosci. 31, 7753-7762

Kim, Y. S., Paik, S. K., Cho, Y. S., Shin, H. S., Bae, J. Y., Moritani, M., Yoshida, A., Ahn, D. K., Valtschanoff, J., Hwang, S. J. et al. (2008). Expression of P2X3 receptor in the trigeminal sensory nuclei of the rat. J. Comp. Neurol. 506, 627-639.

Kim, Y. S., Son, J. Y., Kim, T. H., Paik, S. K., Dai, Y., Noguchi, K., Ahn, D. K. and Bae, Y. C. (2010). Expression of transient receptor potential ankyrin 1 (TRPA1) in the rat trigeminal sensory afferents and spinal dorsal horn. J. Comp. Neurol. 518, 687-698.

Kimmel, C. B., Ballard, W. W., Kimmel, S. R., Ullmann, B. and Schilling, T. F. (1995). Stages of embryonic development of the zebrafish. Dev. Dyn. 203, 253310.

Knaut, H., Blader, P., Strähle, U. and Schier, A. F. (2005). Assembly of trigeminal sensory ganglia by chemokine signaling. Neuron 47, 653-666.

Kohashi, T. and Oda, Y. (2008). Initiation of Mauthner- or non-Mauthner-mediated fast escape evoked by different modes of sensory input. J. Neurosci. 28, 1064110653

Kucenas, S., Soto, F., Cox, J. A. and Voigt, M. M. (2006). Selective labeling of central and peripheral sensory neurons in the developing zebrafish using P2X(3) receptor subunit transgenes. Neuroscience 138, 641-652.

Kyriakatos, A., Mahmood, R., Ausborn, J., Porres, C. P., Buschges, A. and El Manira, A. (2011). Initiation of locomotion in adult zebrafish. J. Neurosci. 31, 8422-8431.

Lee, J. S., Ray, R. and Chien, C. B. (2001). Cloning and expression of three zebrafish roundabout homologs suggest roles in axon guidance and cell migration. Dev. Dyn. 221, 216-230

Light, A. R. and Perl, E. R. (1979). Spinal termination of functionally identified primary afferent neurons with slowly conducting myelinated fibers. J. Comp. Neurol. 186, 133-150.

Liu, K. S. and Fetcho, J. R. (1999). Laser ablations reveal functional relationships of segmental hindbrain neurons in zebrafish. Neuron 23, 325-335.

Liu, Y. and Ma, Q. (2011). Generation of somatic sensory neuron diversity and implications on sensory coding. Curr. Opin. Neurobiol. 21, 52-60.

Luo, L. and Flanagan, J. G. (2007). Development of continuous and discrete neural maps. Neuron 56, 284-300.

Ma, L. and Tessier-Lavigne, M. (2007). Dual branch-promoting and branchrepelling actions of Slit/Robo signaling on peripheral and central branches of developing sensory axons. J. Neurosci. 27, 6843-6851.

Ma, L. H., Punnamoottil, B., Rinkwitz, S. and Baker, R. (2009). Mosaic hoxb4a neuronal pleiotropism in zebrafish caudal hindbrain. PLOS ONE 4, e5944.

Mapp, O. M., Walsh, G. S., Moens, C. B., Tada, M. and Prince, V. E. (2011). Zebrafish Prickle1b mediates facial branchiomotor neuron migration via a farnesylation-dependent nuclear activity. Development 138, 2121-2132.

Marillat, V., Cases, O., Nguyen-Ba-Charvet, K. T., Tessier-Lavigne, M., Sotelo, C. and Chedotal, A. (2002). Spatiotemporal expression patterns of slit and robo genes in the rat brain. J. Comp. Neurol. 442, 130-155.

Marmigere, F. and Ernfors, P. (2007). Specification and connectivity of neuronal subtypes in the sensory lineage. Nat. Rev. Neurosci. 8, 114-127.

Martin, S. C., Marazzi, G., Sandell, J. H. and Heinrich, G. (1995). Five Trk receptors in the zebrafish. Dev. Biol. 169, 745-758.

Martin, S. C., Sandell, J. H. and Heinrich, G. (1998). Zebrafish TrkC1 and TrkC2 receptors define two different cell populations in the nervous system during the period of axonogenesis. Dev. Biol. 195, 114-130.

Masland, R. H. (2004). Neuronal cell types. Curr. Biol. 14, R497-R500.
Masuda, T., Watanabe, K., Sakuma, C., Ikenaka, K., Ono, K. and Yaginuma, H. (2008). Netrin-1 acts as a repulsive guidance cue for sensory axonal projections toward the spinal cord. J. Neurosci. 28, 10380-10385.

Meyer, M. P. and Smith, S. J. (2006). Evidence from in vivo imaging that synaptogenesis guides the growth and branching of axonal arbors by two distinct mechanisms. J. Neurosci. 26, 3604-3614.

Mombaerts, P., Wang, F., Dulac, C., Chao, S. K., Nemes, A., Mendelsohn, M. Edmondson, J. and Axel, R. (1996). Visualizing an olfactory sensory map. Cell 87 675-686.

Nash, P. G., Macefield, V. G., Klineberg, I. J., Murray, G. M. and Henderson, L. A. (2009). Differential activation of the human trigeminal nuclear complex by noxious and non-noxious orofacial stimulation. Hum. Brain Mapp. 30, 3772-3782.

Noma, N., Tsuboi, Y., Kondo, M., Matsumoto, M., Sessle, B. J., Kitagawa, J., Saito, K. and Iwata, K. (2008). Organization of pERK-immunoreactive cells in trigeminal spinal nucleus caudalis and upper cervical cord following capsaicin injection into oral and craniofacial regions in rats. J. Comp. Neurol. 507, 14281440

Ozdinler, P. H. and Erzurumlu, R. S. (2002). Slit2, a branching-arborization factor for sensory axons in the mammalian CNS. J. Neurosci. 22, 4540-4549.

Perl, E. R. (2007). Ideas about pain, a historical view. Nat. Rev. Neurosci. 8, 71

Prober, D. A., Zimmerman, S., Myers, B. R., McDermott, B. M., Kim, S.-H. Caron, S., Rihel, J., Solnica-Krezel, L., Julius, D., Hudspeth, A. J. et al. (2008). Zebrafish TRPA1 channels are required for chemosensation but not for thermosensation or mechanosensory hair cell function. J. Neurosci. 28, 10102 10110.

Sagasti, A., Guido, M. R., Raible, D. W. and Schier, A. F. (2005). Repulsive interactions shape the morphologies and functional arrangement of zebrafish peripheral sensory arbors. Curr. Biol. 15, 804-814

Schoenebeck, J. J., Keegan, B. R. and Yelon, D. (2007). Vessel and blood specification override cardiac potential in anterior mesoderm. Dev. Cell 13, 254267

Sessle, B. J. (2000). Acute and chronic craniofacial pain: brainstem mechanisms of nociceptive transmission and neuroplasticity, and their clinical correlates. Crit. Rev. Oral Biol. Med. 11, 57-91.

Story, G. M., Peier, A. M., Reeve, A. J., Eid, S. R., Mosbacher, J., Hricik, T. R., Earley, T. J., Hergarden, A. C., Andersson, D. A., Hwang, S. W. et al. (2003). ANKTM1, a TRP-like channel expressed in nociceptive neurons, is activated by cold temperatures. Cell 112, 819-829.

Sugimoto, T., Fujiyoshi, Y., He, Y. F., Xiao, C. and Ichikawa, H. (1997a). Trigeminal primary projection to the rat brain stem sensory trigeminal nuclear complex and surrounding structures revealed by anterograde transport of cholera toxin B subunit-conjugated and Bandeiraea simplicifolia isolectin B4-conjugated horseradish peroxidase. Neurosci. Res. 28, 361-371.

Sugimoto, T., Fujiyoshi, Y., Xiao, C., He, Y. F. and Ichikawa, H. (1997b). Central projection of calcitonin gene-related peptide (CGRP)- and substance P (SP)immunoreactive trigeminal primary neurons in the rat. J. Comp. Neurol. $\mathbf{3 7 8}, 425-$ 442

Takashima, Y., Daniels, R. L., Knowlton, W., Teng, J., Liman, E. R. and McKemy, D. D. (2007). Diversity in the neural circuitry of cold sensing revealed by genetic axonal labeling of transient receptor potential melastatin 8 neurons. J. Neurosci. 27, 14147-14157.

Thermes, V., Grabher, C., Ristoratore, F., Bourrat, F., Choulika, A., Wittbrodt, J. and Joly, J. S. (2002). I-Scel meganuclease mediates highly efficient transgenesis in fish. Mech. Dev. 118, 91-98.

Todd, A. J. (2010). Neuronal circuitry for pain processing in the dorsal horn. Nat. Rev. Neurosci. 11, 823-836

Williams, J. A., Barrios, A., Gatchalian, C., Rubin, L., Wilson, S. W. and Holder N. (2000). Programmed cell death in zebrafish Rohon Beard neurons is influenced by TrkC1/NT-3 signaling. Dev. Biol. 226, 220-230.

Woolf, C. J. and Ma, Q. (2007). Nociceptors-noxious stimulus detectors. Neuron 55 353-364.

Xiao, T., Staub, W., Robles, E., Gosse, N. J., Cole, G. J. and Baier, H. (2011) Assembly of lamina-specific neuronal connections by slit bound to type IV collagen. Cell 146, 164-176.

Yeo, S. Y., Miyashita, T., Fricke, C., Little, M. H., Yamada, T., Kuwada, J. Y., Huh, T. L., Chien, C. B. and Okamoto, H. (2004). Involvement of Islet-2 in the Slit signaling for axonal branching and defasciculation of the sensory neurons in embryonic zebrafish. Mech. Dev. 121, 315-324.

Ypsilanti, A. R., Zagar, Y. and Chedotal, A. (2010). Moving away from the midline: new developments for Slit and Robo. Development 137, 1939-1952.

Yuan, W., Zhou, L., Chen, J. H., Wu, J. Y., Rao, Y. and Ornitz, D. M. (1999). The mouse SLIT family: secreted ligands for ROBO expressed in patterns that suggest a role in morphogenesis and axon guidance. Dev. Biol. 212, 290-306.

Zhang, Y., Buchholz, F., Muyrers, J. P. and Stewart, A. F. (1998). A new logic for DNA engineering using recombination in Escherichia coli. Nat. Genet. 20, 123-128.

Zylka, M. J., Rice, F. L. and Anderson, D. J. (2005). Topographically distinct epidermal nociceptive circuits revealed by axonal tracers targeted to Mrgprd. Neuron 45, 17-25 